\title{
Architecture and Intermodality. Guidelines for the Architecture of the Intermodal Hub at the FVG Airport Ronchi dei Legionari
}

\section{Giovanni Fraziano, Thomas Bisiani, Luigi Di Dato, Claudio Meninno, Adriano Venudo and Marko Verri}

\begin{abstract}
The research addresses the topic of intermodality in terms of technical, typological, and architectural response of infrastructure systems, considering their relationship with the territory and the landscape. The study starts from the hypothesis of the development of a comprehensive intermodal hub in the Ronchi dei Legionari Airport (Friuli-Venezia Giulia, Trieste, Italy), integrated with the network of local public transport and railway infrastructure, considered in a scenario of sustainability over the medium term and updated with respect to the current economic situation. The first phase of the research focused on the definition of the guidelines for the design of the intermodal hub. Subsequently, the study verified the hypothesis by modeling some alternative scenarios, whose results led to the identification of a highly comprehensive set of data, consistent with the financial framework. Lastly, urban verification: the identified scenarios constitute a framework of alternative options corresponding to the minimum, optimal and critical conditions of the system as a whole. The possible alternatives prove the degree of versatility of the proposed layout which provides, as a whole, the possibility of opting for one of the proposed scenarios or for a more complex combination of the proposed solutions.
\end{abstract}

Keywords: intermodal hub, infrastructure, evolutionary scenarios, strategic planning, Friuli Venezia Giulia

The development of an "application" model of sustainable intermodality at different levels, local and regional. Complete and coherent under different profiles: architectural, urban and territorial, infrastructural, 
economic, managerial and organizational. It is the final outcome of the operational research that has led, through a two-year commitment, to the definition of the guidelines necessary for the architectural configuration of the Intermodal Hub annexed to the FVG Ronchi dei Legionari Airport.

The research, the results of which have been widely recognized at the national and international level, has proved to be fundamental for the development of the final design of the Hub and therefore for the subsequent implementation phases. The term "operational," in this experience, applies to the influence and the direct impact of a process of knowledge and formulation of the project, divided into analytical, interpretative and technical verification phases, avoiding any insistence and/or entrenchment in the definition of abstract models, or in selfserving logic.

The study of the material context and the territorial systems, with particular reference to urban, landscape, environmental, hydro geological and airfield statutory constraints was followed by analysis of the infrastructure system and the main connected networks. The study of flows and relations with the different territorial dynamics affected by the Intermodal Hub. The assessment of costs and benefits attributable to the more relevant modality model, the definition and comparison of alternative layouts. The dimensioning of a functional executive program in relation to policy orientations aimed at forecasting the development of the FVG Airport in terms of growth of flows and extension of the user base as well as the confirmation of the same in its role of strategic infrastructure for the regional context.

Hence, the technical and regulatory pre-dimensioning of areas, structures, roads, car parks, technological artifacts, open spaces and formal devices. The feasibility assessments and performance verifications related to the capacity and service level of the infrastructure and annexed elements.

The simulation, or simulations, related to the architectural configuration of the parts and the elements constituting the complex whole. A second phase concerned the updating of the analyses and the territorial frame of reference as well as the configuration of transformation scenarios, in the medium and long-term: Reversible minimum scenario, Optimal scenario or Standard Service Level and Crisis Scenario with the identification of the maximum sustainability potential of the infrastructure.

The definition of the guidelines, in essence the creation of the "rules of the game," was not, as it might seem looking at the chronological listing of the various phases, an arid and exhausting crossing of data with a reductionist logic, but rather the attempt to address the complexity and the importance of a topic that is multifaceted and rich in implications such as an infrastructure, and more generally the theoretical and experimental steps concerning the architectural and landscape design connected to such infrastructure, by testing tools and techniques, disciplines and economies, fields of study, practices, and even testing 


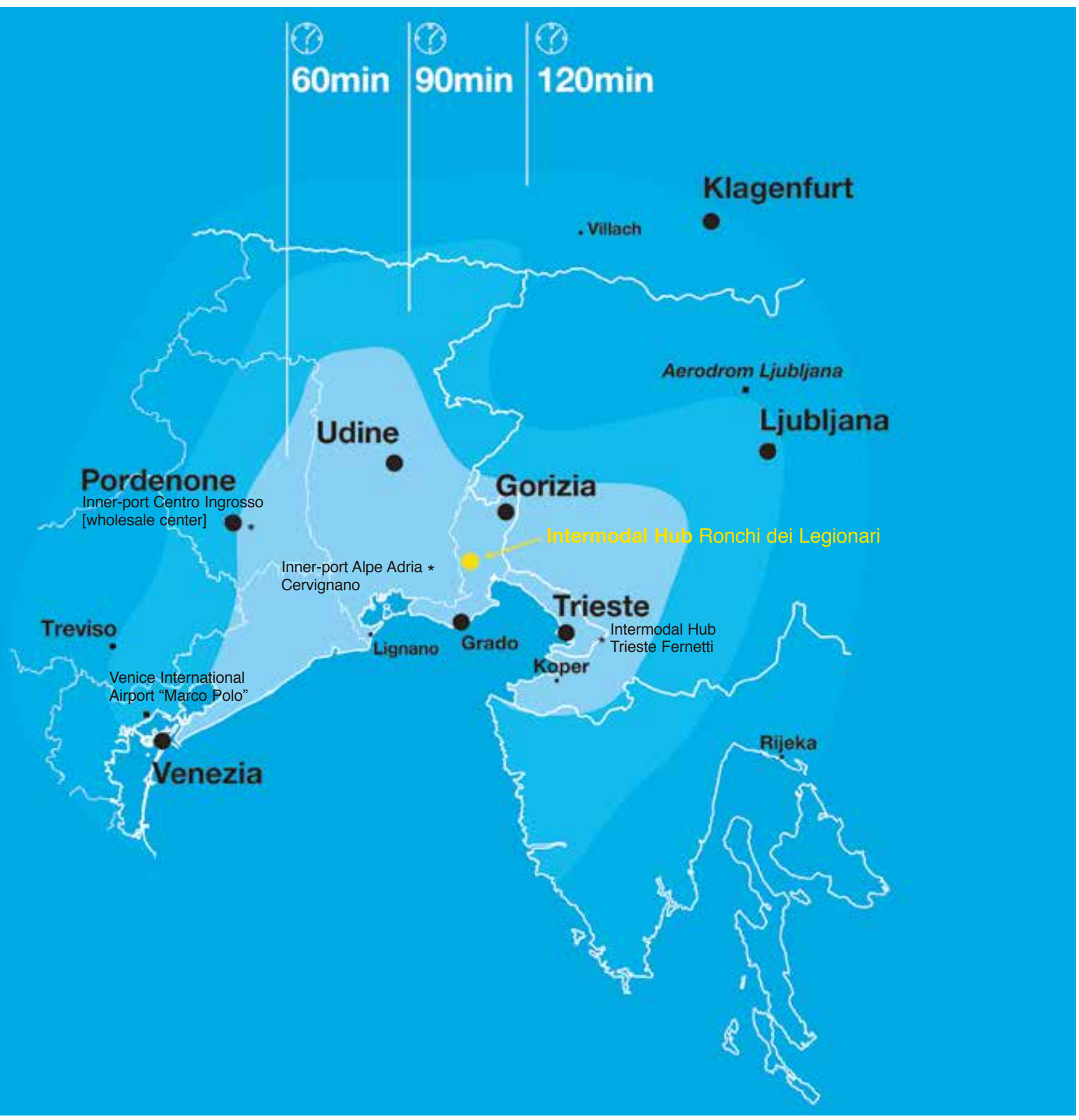

Figure 1. Macro area of the Intermodal hub adjacent to the Ronchi dei Legionari airport.

the authors, who are of different ages and have different skill sets, in a truly multi-disciplinary action.

An action that, with different technical approaches and expressions, leads to a synthesis of the study, reflection and exploration phases, and to a method that illustrates the entire scope of the project and that can be understood, interpreted and revisited. This value of general applicability represents a major academic achievement of this research, and it represents and constitutes a high expression of design capability. 


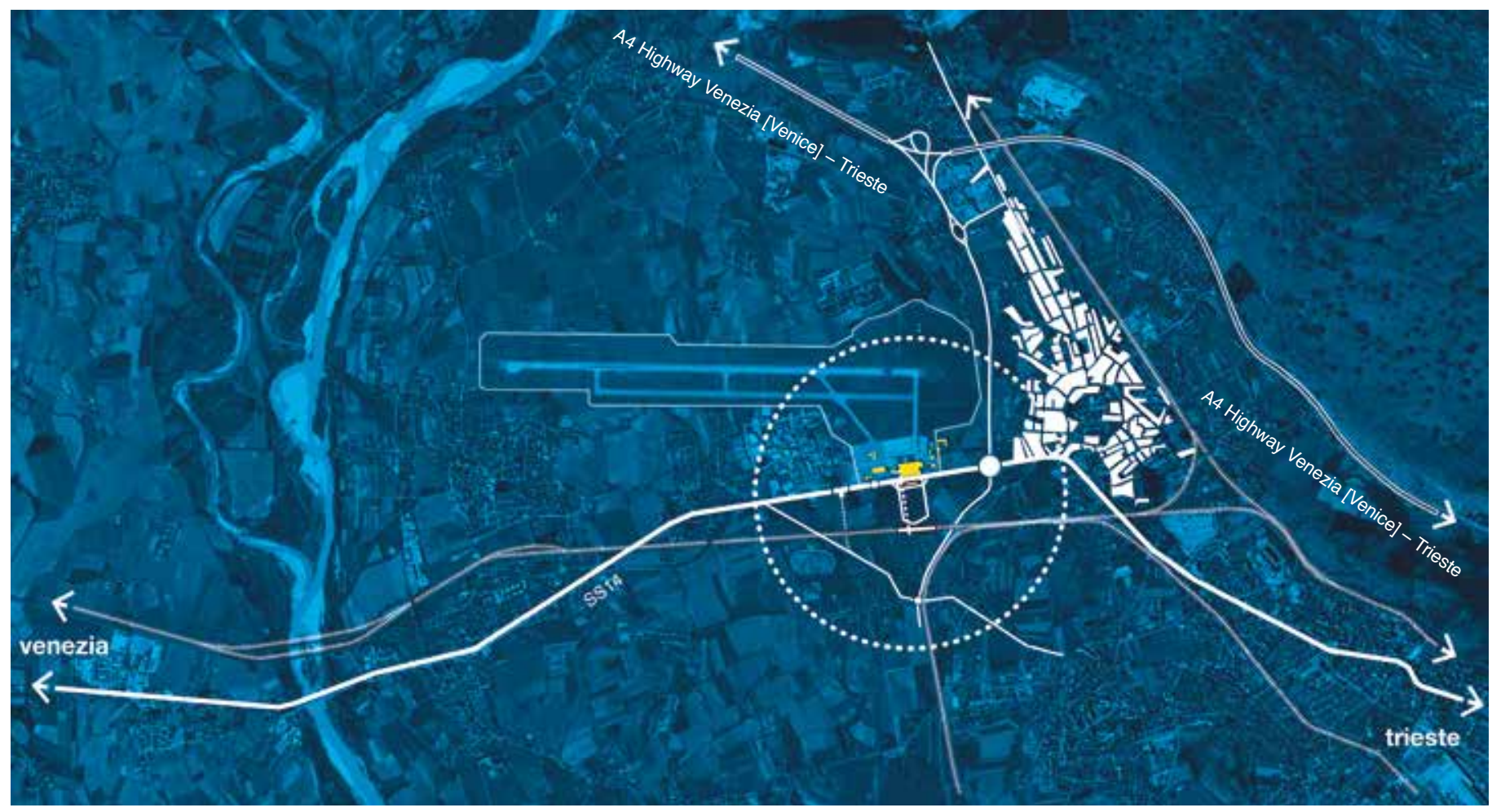

Figure 2. Micro context of the Intermodal hub including the Ronchi dei Legionari airport.

\section{Research Program}

The research includes the examination of the intermodality topic in terms of technical, typological and architectural response of the infrastructure systems, considering their relationship with the territory and the landscape.

Specifically related to the operational dimension, the research starts from the acquisition of a model of international scope comparable to the size, traffic flow and complexity of the regional airport of Ronchi dei Legionari, considered together with the annexed area as an object of study and a privileged field for experimentation and synthesis.

Divided into steps, the research leads to the definition of guidelines useful for the design of the intermodal exchange centre and the airport complex, with a high conceptual and qualitative profile, and to the configuration of an appropriate economic and functional feasibility, also considering sustainability aspects and environmental balance implications.

\section{Hypothesis of the Study}

The research has started from the hypothesis of the development of an intermodal hub connected to the Ronchi dei Legionari airport and integrated with the local road and rail public transport infrastructure, with reflections at the international level. The whole project has been 


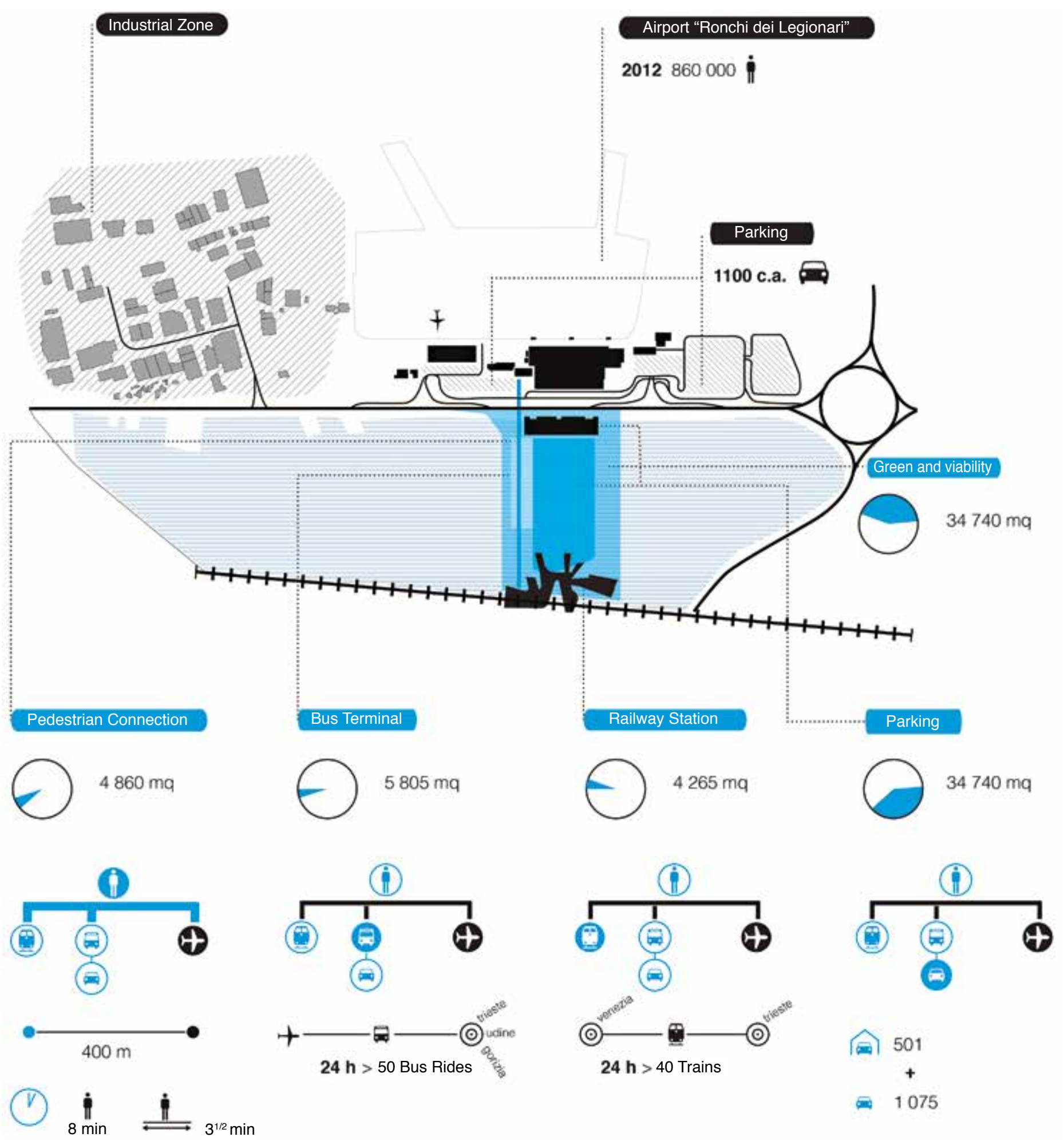

Figure 3. Master plan info-graphic. 
considered within a broader sustainability framework of the interventions in the medium term, updated to the current economic situation.

In particular, the intention has been to explore the study of the intermodal hub in depth starting from a given functional programme, as a defined architectural and infrastructural organism, subsequently broadening the investigation to the possible urban articulations of the connected surrounding areas.

The main connection axis was identified in the elevated canopy that is connected with the various functions of the Intermodal Hub, integrating certain functions of individual parts in order to decrease the number of functional elements, to optimize the necessary economic resources and strengthen the identity of the architectural setting. In particular, in the railway station the canopy becomes the overpass of the tracks and in the bus station it becomes the roofing of the lower space. In this way, only a few other elements are necessary for the definition of the spaces, with obvious scale benefits.

\section{Methodology Used}

The general methodology used to develop the project hypotheses provides for two distinct types of activities: The first is the analysis of the context, carried out based on the various aspects being considered relevant or distinctive; the second phase assumes that this analysis is then verified by constructing scenarios aimed at providing alternative solutions to the contextual framework previously defined. In the study, these alternative scenarios have been compared to each other in order to determine the optimal solution and define the guidelines for the object of the research.

In particular, this methodology has been applied at different levels, urban and architectural, in order to take into account both the overall arrangement of the hub and the most relevant and sensitive architectural solutions.

\section{Analyses Conducted}

Urban context: a screening of the existing planning instruments at the different territorial levels has been conducted in order to acquire an overall picture of the local and territorial planning concerning the object of the study, aimed at determining compatible scenarios.

Restrictions: The study has identified the restrictions in the area from different points of view, and in particular regarding infrastructure, environment and landscape, in order to determine the "invariant" aspects for the comparison of different scenarios.

Infrastructural context: also with regard to the planning framework, the infrastructural context of the study has been determined in order to create a coherent whole, aimed at the proper inclusion of new flows arising from the verification scenarios. 
Economic context and financial planning: the identified scenarios have been verified with particular attention to the economic and financial aspects, in particular, the short-term feasibility of the Intermodal Hub facilities.

\section{Verification}

The verification of the hypotheses made by the study was carried out by modeling some alternative scenarios, at the urban and architectural level. The comparisons and verifications of different levels have led to different applications of the described study methodology.

Depending on the degree of detail reached, in particular regarding the architectural level and in relation to the Intermodal Hub, it has been possible to identify a more defined and detailed scenario, coherent with the data framework, in particular with financial data. This has allowed us to recognize, among the evaluated alternatives, a single proposal as the solution to be favored.

Subsequently, we proceeded with the urban verification; the overall scenarios identified at this level provide a set of alternative options, defined by the degree of compatibility with the Intermodal Hub solution described above and taken as the invariant element for this broader hypothesis.

\section{Definition of Alternative Scenarios}

For the Intermodal Hub, characterized by a unified functional program defined in agreement with the airport, the hypotheses produced by the study investigated the different spatial and infrastructural configurations that were compatible with the analysis frameworks and suited to minimize the impacts and costs of the works.

Consequently, the study defined different characterizations for the surrounding area that are compatible with three different regimes and intensity of use of the Hub: a minimal condition, an optimal condition and a crisis of the system as a whole.

\section{Identification of the Optimal Solution}

The optimal configuration of the Intermodal Hub has been first identified through an assessment of the various structures, using a parametric quantification of the interventions, then through a detailed analysis of the identified solution with particular reference to construction costs. The surrounding urban structure was assessed in relation to the infrastructural capacity of the Hub, verifying the possibility of developing the surrounding areas with limited adaptations of the Hub itself and the bordering road networks.

To this end, the limit assumed was an infrastructural configuration of the Hub that would not require major works on the bordering national and provincial roads. 


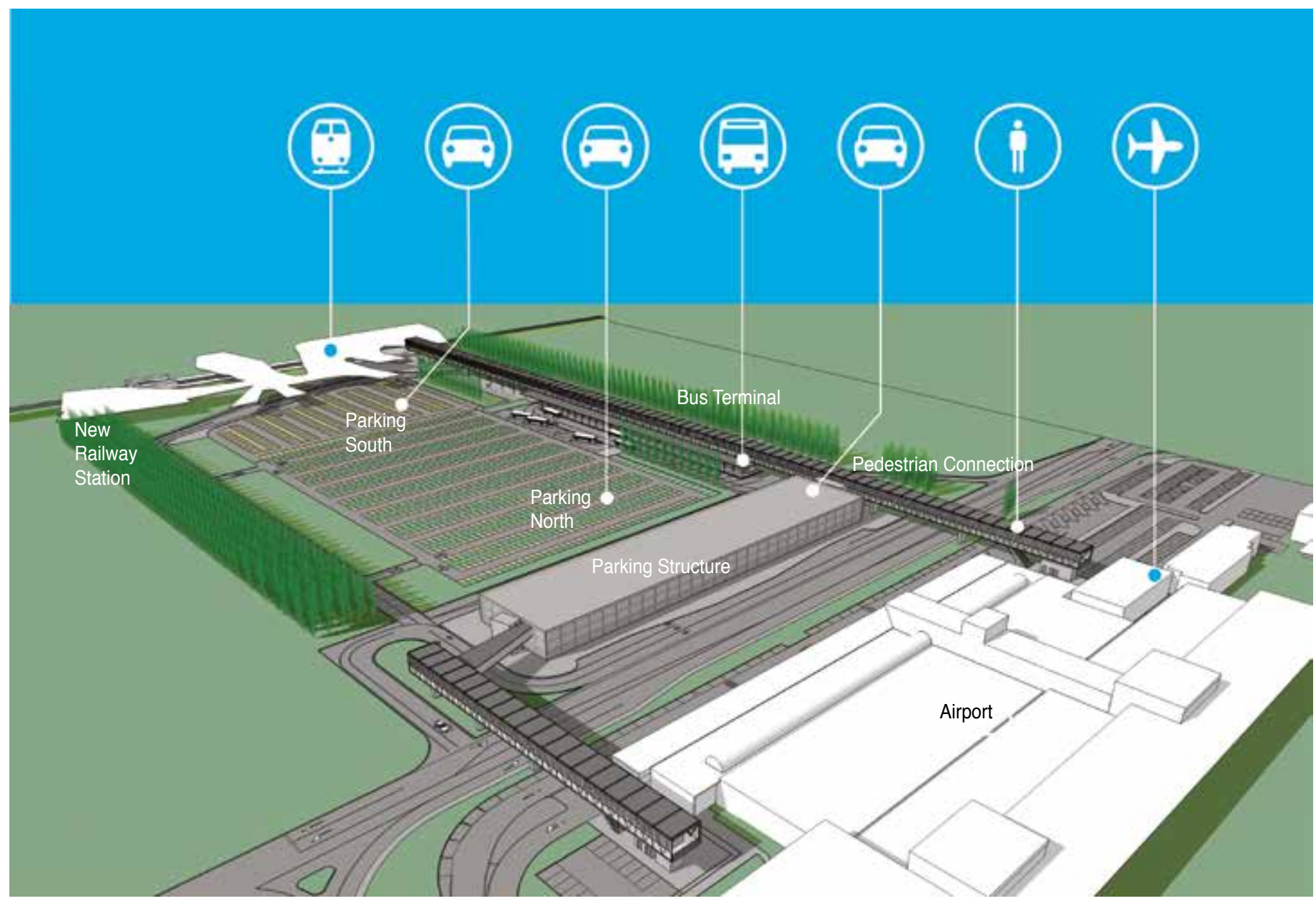

Figure 4. 3D view of the intermodal hub including new functions.

Achieved Results

The achieved results demonstrate the technical and economic feasibility of a first development phase of the Intermodal Hub in the short and medium term, compatible with the analytical frameworks evaluated.

Subsequent scenarios of urban development also confirm the possibility to build on this first element in a sustainable and effective way, without the need to intervene in the existing road system. The possible alternatives that have been identified also demonstrate the degree of versatility of the proposed configuration, which provides the possibility, as a whole and both in the medium and long-term, to opt for one of the proposed scenarios or for a more complex configuration resulting from a structured combination of the solutions offered.

\section{MINIMUM REVERSIBLE SCENARIO}

This hypothesis should be viewed considering the national geopolitical scenario, the regional economy and the local urban settlement. The 


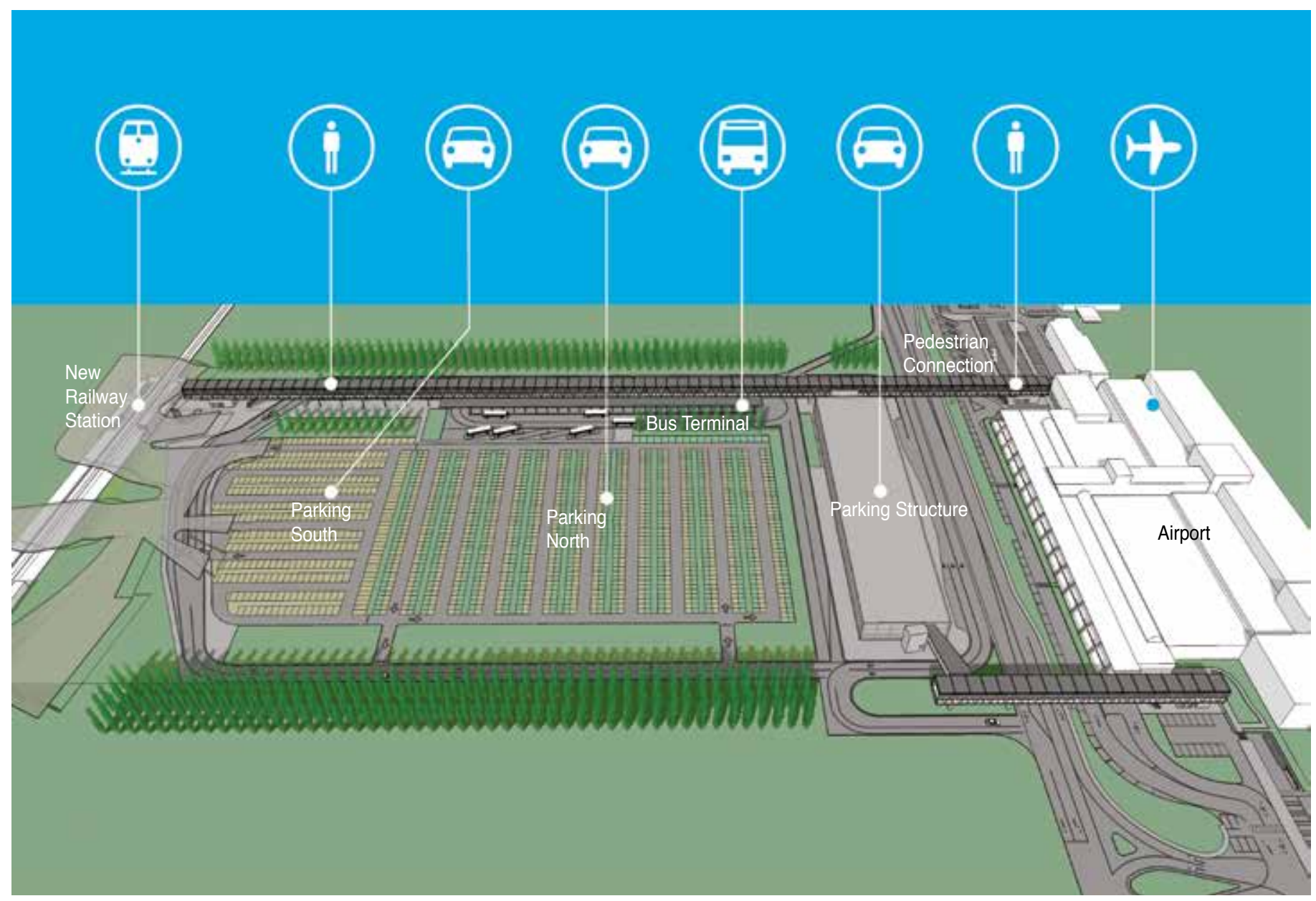

Figure 5. 3D view of the intermodal hub including new functions.

proposed layout provides for a minimal reorganization of the area with a different use of agricultural lands, characterized by a low environmental impact and reduced use of resources for infrastructural works. The agrarian vocation of the area is confirmed, with experimentations in specialized sectors such as intensive micro-agriculture, bio-agriculture or multifunctional agriculture (as called for by the recent feasibility study for the "Experimental Landscape Plan of the Province of Gorizia"), while waiting for a reversal of the declining trend of the regional economic scenario and the local production. The scenario explores a possible development of the East and West Sectors in line with the theory of "de-growth."

All infrastructure and urbanization interventions have a reversible nature. The scenario provides for a road system with a backbone that makes use of the ring road of the Intermodal Hub.

The service level of the Sectors is guaranteed and integrated by the service level of the Intermodal Hub that, in relation to the trend of the flows related to the Airport and those planned from the new RFI stop and the CIMR bus line, offers a wide margin of functionality. 
The scenario envisions an internal operation of the Sectors (urban and infrastructural) that exploits the time needed for the full operation of the Intermodal Hub (the estimate is at least 5 years). This, therefore, is a short-term scenario, but it does not necessarily constitute a preparatory phase for future transformations.

\subsubsection{General Choices, Objectives, Strategies and Overall Vision}

The scenario provides for a minimal reorganization of the area with the creation of a non-food agricultural system. The reorganization of existing lands has sought to minimize the changes to the existing situation, in order to streamline the implementation procedures, by identifying two Sectors, West and East, characterized by a reversible infrastructural system with a low environmental impact, which requires limited resources for its implementation.

Starting from an approach in line with the theory of "decrease" and requiring a limited land use, the identified scenario appears as a short-term option, capable of absorbing and facilitating the evolution towards other scenarios with a more intensive usage but not being necessarily only a preparatory phase for future transformations.

\subsubsection{Settlement Model}

When investigating the optimal settlement model, the analysis has taken into account various aspects related to the present situation and the future Intermodal Hub. First of all, the study has tried to maintain the current land situation as much as possible, identifying a distribution layout capable of providing maximum functionality and requiring minimum economic and bureaucratic efforts, so as to facilitate the implementation procedures; secondly, it has tried to minimize the impact of the main road system exploiting the existing or planned routes. Consequently, the creation of the East and West Sectors can be implemented with a significant reduction in costs and construction times.

\subsubsection{Road System and Distribution}

The distribution road system is identified with a precise layout and provides for changes of the road platform based on the scenarios that have been identified by the research. This, together with the re-use of almost $80 \%$ of the ring road of the Intermodal Hub, enables the containment of the implementation and development costs for the entire road system, which consists of several roads of various types linked by a precise hierarchy: a backbone that branches off from the ring of the Intermodal Hub, a ring that defines and surrounds the South Sector, a system of penetration roads and a bike path connected with the existing local cycle network.

The entire road system has been developed in parallel to a landscape 


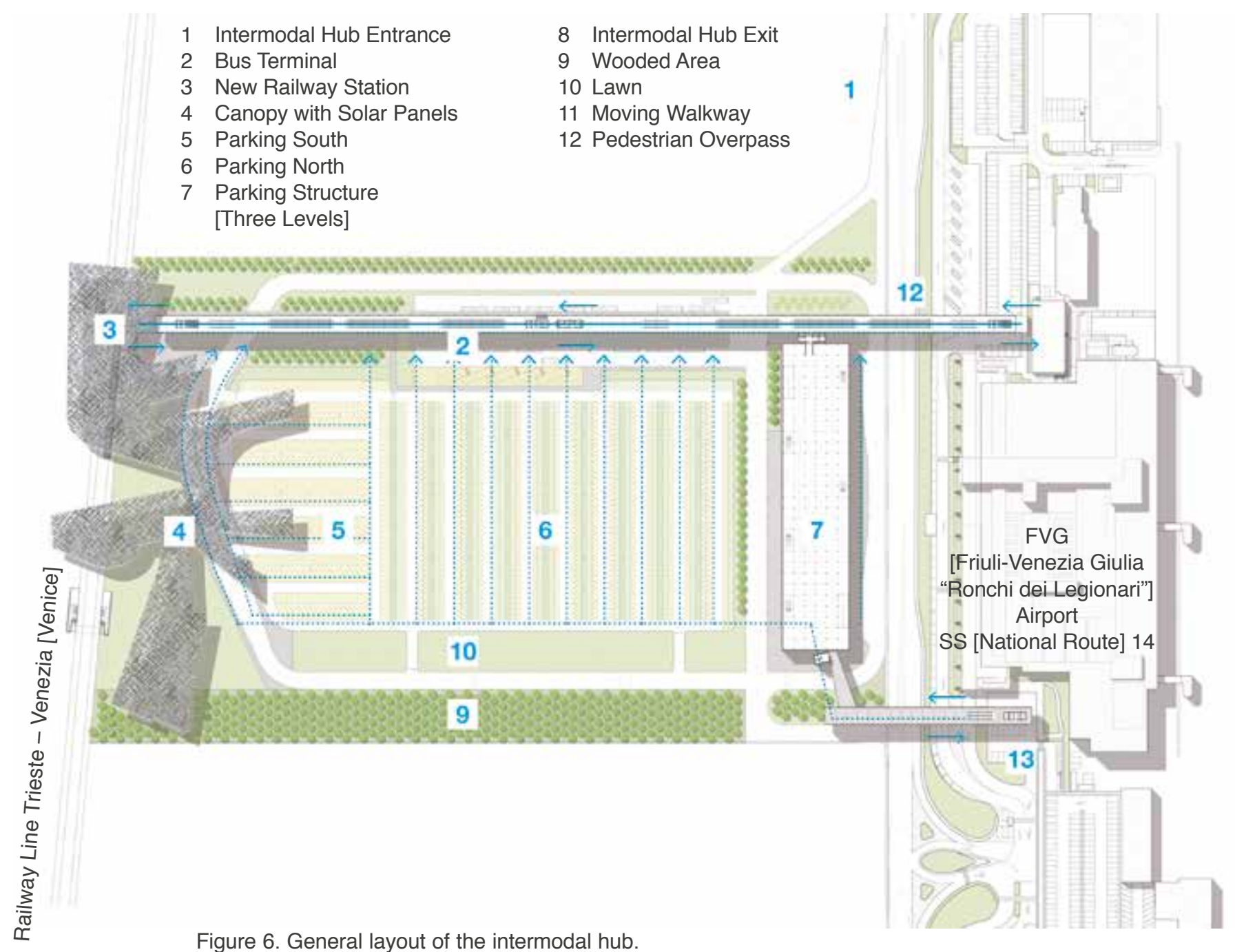

Figure 6. General layout of the intermodal hub.

project, having as its main elements rows of trees along the penetration roads, green buffer zones and a wooded diaphragm towards the railway.

\subsection{Settlement Models}

\subsubsection{Main Functions, Induced Uses}

The primary use that characterizes this scenario is of the non-food agricultural type. The non-food specification depends on the fact that the area is affected by the presence of exhaust gases from the airport that are incompatible with food crops.

The development of the agricultural activity in the area can be divided, as better explained in the following diagrams, into sub-scenarios, which involve the use of the area mainly for field cultivation, greenhouses, a mixed scenario and, finally, intensive exploitation. In addition to the agricultural component, the Minimum Reversible 
Scenario provides for the creation of a park/diaphragm close to the railway, a green buffer area and another area, located within the South Sector, for a possible expansion of the Intermodal Hub.

\subsubsection{Levels of Flexibility and Possibility of Modification}

The entire system identified, according to different phases and modulations, ensures a high degree of flexibility and the possibility of modification both with respect to the evolutions of the other scenarios, through the continuation of the settlement scheme and the maintenance of the road system design, and within the Minimum Reversible Scenario with the possibility of establishing and implementing agricultural activities with different types of land use.

\subsubsection{Land Use, Density, Green Areas, Economical Convenience and Pragmatism}

Land use has been minimized through the creation of a lean distribution system and adopting solutions with a low environmental impact. The green areas are used as buffer zones between the cultivation zones, the road to the terminal and the rail movement axis. The economic convenience of the design choices has already emerged from the identification of the guidelines of the settlement model with the motivations provided above.

\subsection{Urban Layout and Contextualization of the Scenario}

\subsubsection{Relations with the Intermodal Hub}

The relationship with the Intermodal Hub takes place through the interdependency with the distribution ring of the multi-modal system, the creation of an area for possible expansion in the West Sector and the development of a cycle system that, connecting with the existing dedicated networks, provides the possibility of implementing and further enhancing the interchange within the area affected by the general intervention.

\subsubsection{Connections to the Context}

The connections to the context occur through the integration with the existing infrastructure, with the Intermodal Hub and with the airport, while from a landscape point of view there are common and recognizable elements of the context in which the scenario in question is meant to operate.

\subsection{Conclusions}

The Minimum Reversible Scenario represents a very satisfactory answer to the current and future minimum requirements and larger potential. The hypothesis identified is configured as a serious and sustainable scenario from both an environmental and an economic standpoint, providing the 
Figure 7. View from the parking lot.

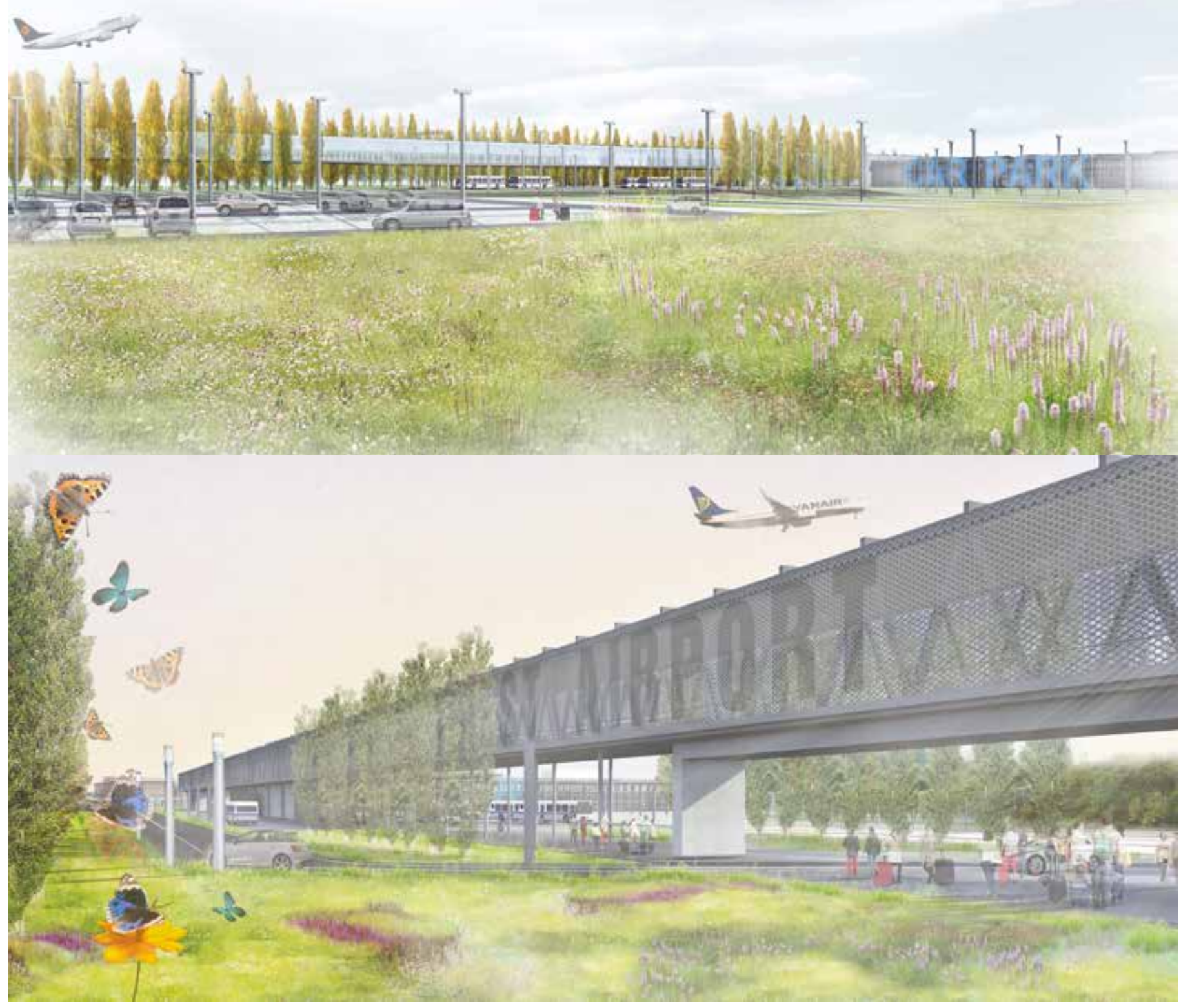

Figure 8. View of the footbridge.

possibility for further development towards optimal or crisis scenarios through moderate implementations of the settlement model.

\section{OPTIMAL SCENARIO OR STANDARD SERVICE LEVEL}

This hypothesis assumes a scenario of national and European geopolitical growth and a regional framework of rationalization of production activities 
Figure 9. Optimal scenario, master plan.

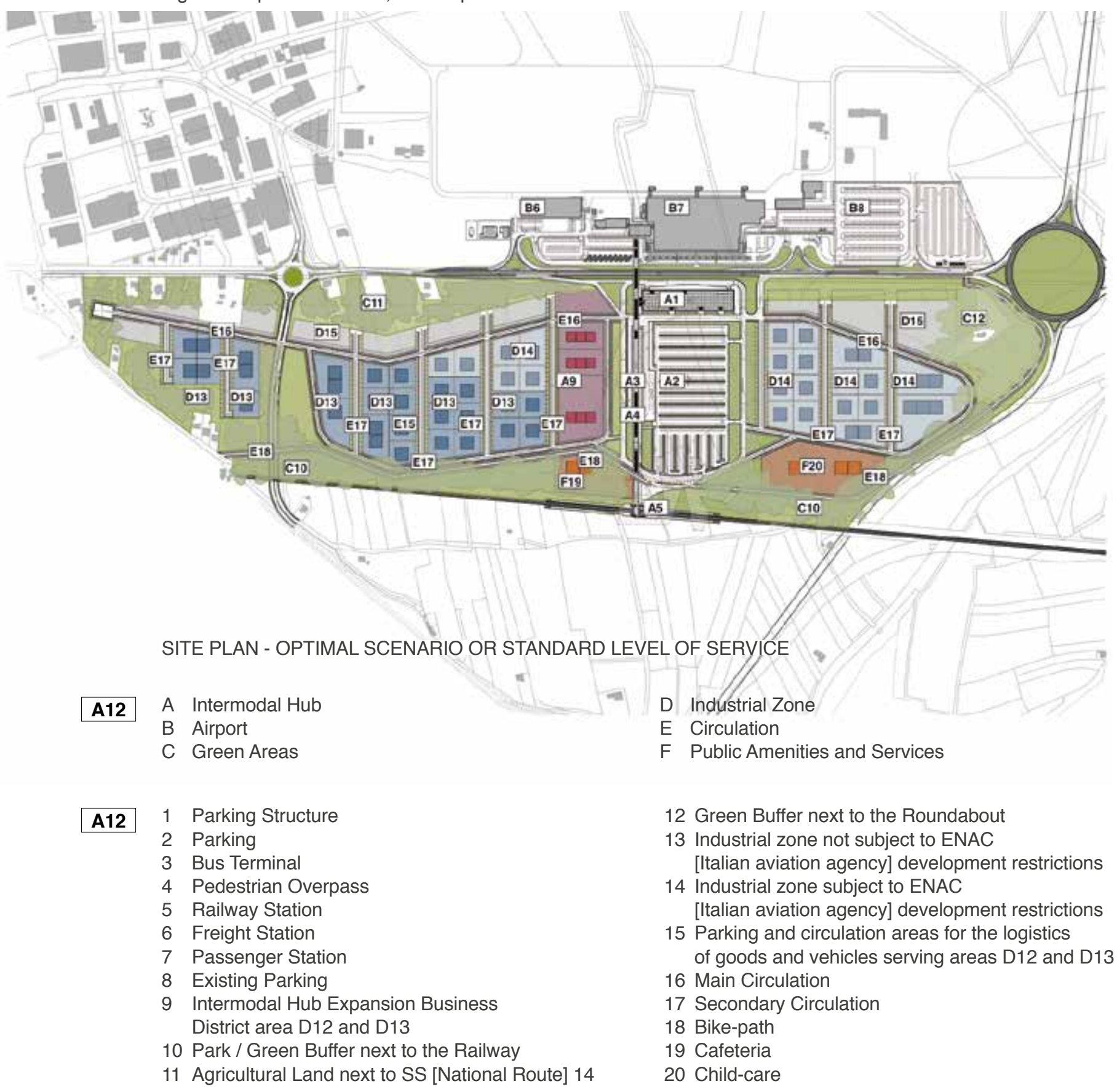

and urban reorganization carried out by local administrations (provinces and municipalities). Regional Law 5/2007, as amended, provides for several administrative cooperation instruments, including the integrated management of municipal production areas with a perspective of land use optimization. Starting from these assumptions, the Sectors create a substantial, comprehensive and well-equipped manufacturing/production area, with a strong environmental character (APEA), fuelled by the new "regional environment exchange platform" in service of all municipalities in the District. 
The hypothesis is based on the equalized transfer of " $\mathrm{D}$ " areas of the 9 municipalities of the District to Sectors adjacent to the Intermodal Hub, with the related transfer and compensation of expenses. In this way, the Sectors would be configured as a production district with a strong critical mass, a high level of specialization/experimentation and a wide range of spaces, infrastructure and equipment, and above all with access to major networks and logistics routes. The structural elements (invariants), in addition to the road system, will consist of the environmental system, which will characterize the ecologically equipped production area. The scenario offers an "ideal" level of service (TGM 2600/2800) as it leverages the full implementation of the Hub and increases its capacity with the addition of a dedicated ring on the side of the ring of the Intermodal Hub. The two Sectors will be structured in two rings connected to the middle one, enlarged, of the Intermodal Hub, and a widespread grid system, within the rings, which will organize the layout of the settlement model.

\subsubsection{General Choices, Objectives, Strategies and Overall Vision}

The Optimal Scenario of the study envisions the possibility of creating an intermunicipal manufacturing/production district compatible with the constraints of the area deriving from the presence of the airport. The implementation can be carried out directly or as an evolution of the Minimum Reversible Scenario and provides a wide modulation of the structure and volume requirements of the various production units that may be established. Considering the location of the area in relation to its surroundings, the district can be either an implementation of existing neighboring entities or as a consolidation and optimization of micro-districts existing in a larger surrounding area. In this way, a virtuous circle could be triggered that optimizes existing resources and potentials, simultaneously converting low-value manufacturing/production areas currently present in the surrounding municipalities. In this sense, the Optimal Scenario is proposed as a solution not only for the East and West Sectors, but also for a much larger area that have in the airport and in the future Intermodal Hub an important development factor.

\subsubsection{Settlement Model}

The settlement model of the Optimal Scenario follows what has been described in relation to the Minimum Reversible Scenario. Its implementation is based on the same assumptions, i.e. the optimization of resources using local assets as a design input in order to align the reduction of implementation costs and timeframes with the creation of a scenario able to provide optimal responses to present and future needs. The system can be accomplished either directly or as an evolution of the Minimum Reversible Scenario. In this case, it will be sufficient to re-modulate some roadways and create the necessary urban infrastructure for the settlement of the various manufacturing/production activities. The settlement model, finally, can evolve towards the requirements of the Crisis Scenario. 


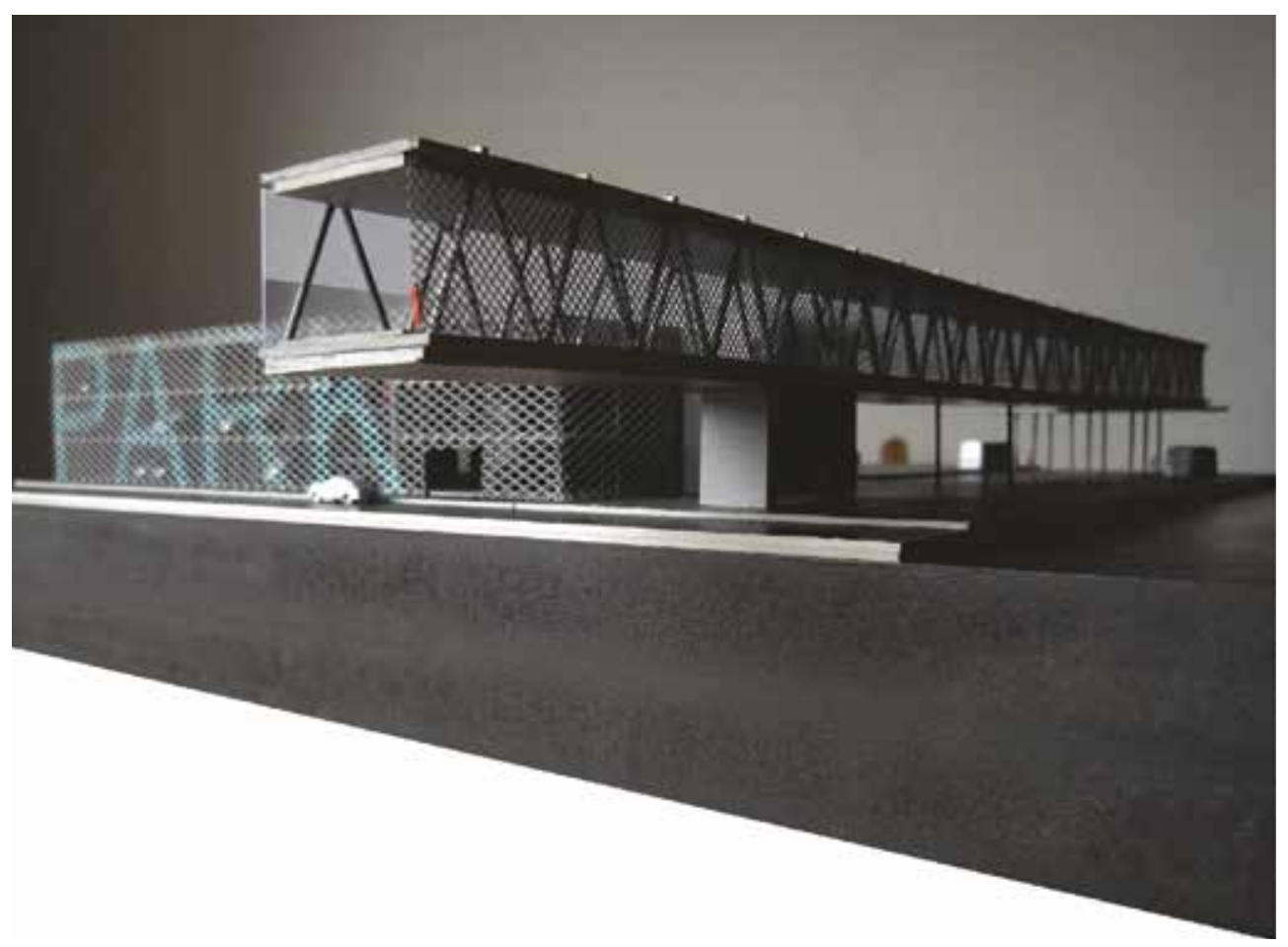

Figure 10. Detail of the mock-up zooming on the new footbridge.

\subsubsection{Road System and Distribution}

Maintaining the general principles of the road system for the Minimum Reversible Scenario, in this case the study provides for the creation of a hierarchical road system with roadways calibrated according to the specific needs arising from the presence of manufacturing and production units. The overall design identifies a main backbone complete with a circulation ring that defines the area destined to the future expansion of the Intermodal Hub, two rings for the circulation in the East and the West Sectors, a penetration road system along the a North-South axis and finally a cycle path that, developed in the South part of the area, connects with the existing cyclepedestrian system. The road system is accompanied by a landscaping project that maintains the distinguishing features already present in the Minimum Reversible Scenario, like the tree-lined avenues along the NorthSouth axis and the wooded diaphragms towards the rail and road systems that surround the area concerned by the study.

\subsection{Settlement Models}

\subsubsection{Main Functions, Induced Uses}

The activities that will be established will be connected to a manufacturing and production model. According to the identified subscenarios, there will be a different densification of the area and of the 


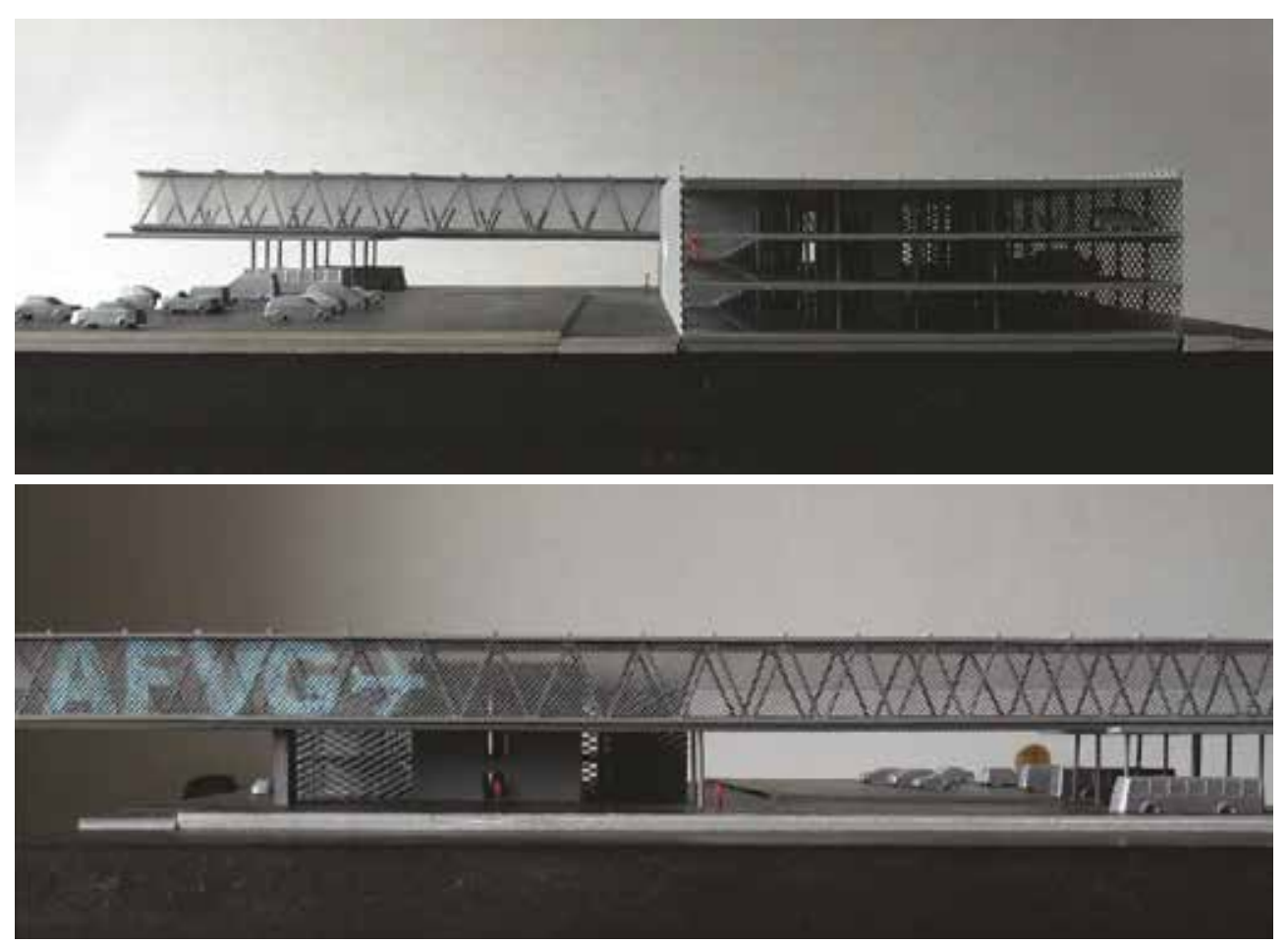

Figure 11. Views of the project mock-up.

cubic volume of the buildings. The smaller units will be $20 \times 20 \mathrm{~m}$ on a single lot, larger units may result from the merging of a number of minimum units plus a premium allotment. The north part of both Sectors will have large areas dedicated to parking lots and cargo handling. An area dedicated to the expansion of the Intermodal Hub will be identified inside the West Sector. While in the vicinity of the park/ diaphragm near the railway the study envisions complementary functions such as a canteen and a kindergarten in order to increase the service level offered to all the workers within the area and its immediate vicinity, all in full compliance of Enac and rail constraints. In addition to the aforementioned green area, additional green areas will be identified as buffers towards the major arterial roads.

\subsubsection{Levels of Flexibility and Possibility of Modification}

The system identified, with the related land layout, provides the possibility to create highly flexible production areas, organized according to a mesh that determines minimum production units and a number of progressive variations, until reaching a sub-scenario of Maximum Cubic Volume. The manufacturing/production areas are divided into two sub-areas according to the need to comply with the ENAC constraint on a massive presence of people. The presence of parking and handling areas completes a service already made strategic by the presence of the Intermodal Hub. 


\subsection{Urban Layout and Contextualization of the Scenario}

\subsubsection{Stakeholders and Investors}

This hypothesis assumes a scenario of national and European 2020 growth and a regional framework of rationalization of production activities and urban reorganization carried out by local administrations (provinces and municipalities). Regional Law 5/2007, as amended, provides for several administrative cooperation instruments, including the integrated management of municipal production areas with a perspective of land use optimization. Starting from these assumptions, the Sectors create a substantial, comprehensive and well equipped manufacturing/production area, with a strong environmental character (APEA), fuelled by the new "regional environment exchange platform" in service of all municipalities in the District, and therefore it will be attractive for both new activities and for activities coming from neighboring municipalities. This will happen thanks to the equalized transfer of "D" areas of the 9 municipalities of the District to Sectors adjacent to the Intermodal Hub, with the related transfer and compensation of expenses. In this way, the Sectors will constitute a production district with a strong critical mass, a high level of specialization/experimentation and a wide range of spaces, infrastructure and equipment, and above all access to major access to major networks and logistics routes.

\subsubsection{Land Use, Density, Green Areas, Economical Convenience and Pragmatism}

The overall system includes the optimization of the road system for a ratio between usable areas/available roads very much in favor of the first. In this way, it will be possible to rationalize the use of space and there is the possibility of achieving a densification of the built volumes, optimizing land use. The overall design identifies lots which are as regular as possible so as to avoid waste and concentrates parking and handling spaces in the perimeter areas, achieving a further rationalization of the flows and the use of the area.

\subsubsection{Relations with the Intermodal Hub}

The entire manufacturing/production area benefits from the presence of the Intermodal Hub thanks to which its offer is enriched by a unique exchange system and vehicular communication in the region, with obvious benefits in terms of logistics. This, together with the possibility of synergistic relationships between the activities that will be established in the area, makes the subject of the study particularly attractive and an ideal candidate as one of the leading sites for the future economic recovery of the entire region. 


\subsubsection{Connections to the Context}

The connections to the context are made evident by the integration with the Intermodal Hub, with the airport and with the existing and planned road and rail systems. From a landscape point of view, the study has developed green areas that integrate the intervention and put it in relation to the characteristic elements of the surrounding landscape, the access to which is also facilitated by the presence of a bicycle path connected with the bicycle and pedestrian system already existing in the area.

\subsection{Conclusions}

The Optimal or Ideal Standard Scenario is a constructive and concrete answer, both in case of economic recovery of the entire area and in the hypothesis of optimization and incorporation of the various micro-districts currently present in the municipalities of the District, according to a sustainable approach from an environmental and economic standpoint. The proposed scenario can be configured as an evolution of the Minimum Sustainable Scenario or as a direct implementation. In turn, it provides the possibility of an evolution towards a Crisis Scenario through some modification to the layout of the premises, i.e. exclusively eliminating the penetration roads from the main system.

\section{CRISIS SCENARIO AND IDENTIFICATION OF THE MAXIMUM SUSTAINABLE SERVICE LEVEL}

\subsubsection{General Choices, Objectives, Strategies and Overall Vision}

The scenario is set in a context of growth and economic recovery at various levels (regional and local).

The urban and infrastructural operation follows the "ideal standard" scenario, leveraging the Intermodal Hub as an entry/exit point of the system, and serves the Eastern and Western sectors with high-capacity one-way internal loops (3000/3200 TGM).

The hypothesis investigates the possibility of maximum saturation of the area up to the limit of the service level provided by the Intermodal Hub. To go beyond that, would require a total revision of the model and the layout of the roadway system constituted by the roundabout towards Grado, the airport section of the SS14 and the new roundabout on the SS14 in Begliano. Through several traffic administration and rationalization instruments, it will be possible to use the road network at maximum capacity, in order to ensure maximum settlement density. Beyond that, the system would go into a crisis (congestion and blocking). The study provides for a mix of functions that integrate manufacturing/production activities, logistics and "differentiated logistics," the City Port systems (servicing the District, but also Trieste and Gorizia) and Eco-Logistics, in addition to services and complementary activities supporting the main activities. 
Figure 12. Crisis Scenario, master plan.

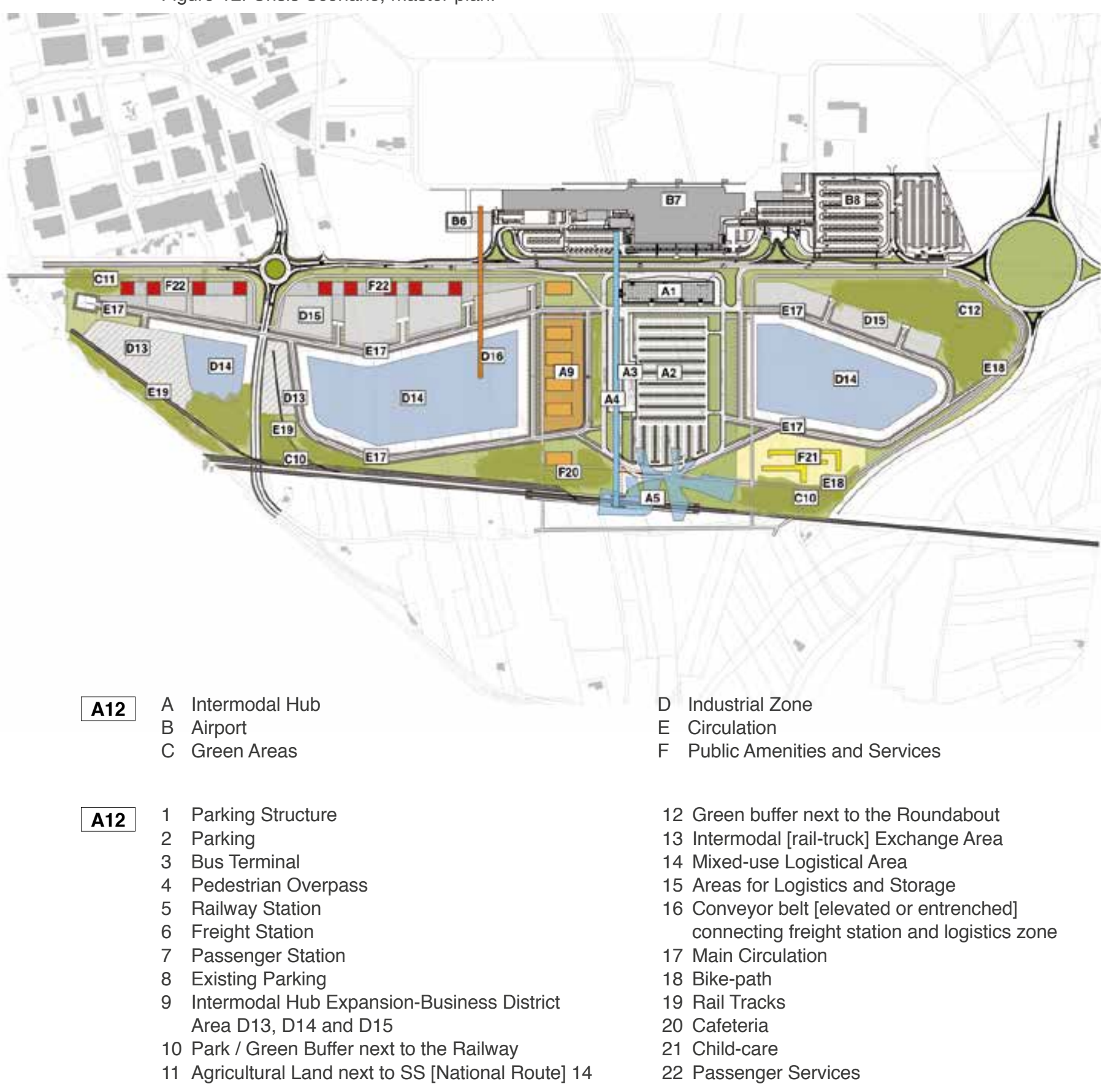

\subsubsection{Settlement Model}

The general structure behind the Crisis Scenario represents the evolution of the Optimal Scenario, following the settlement model illustrated above, with the difference that the penetration roads are eliminated to allow further development of the built areas, with a greater volume capacity that can accommodate production and logistics functions requiring large operational volumes. 
Figure 13. Minimum reversible scenario, master plan.

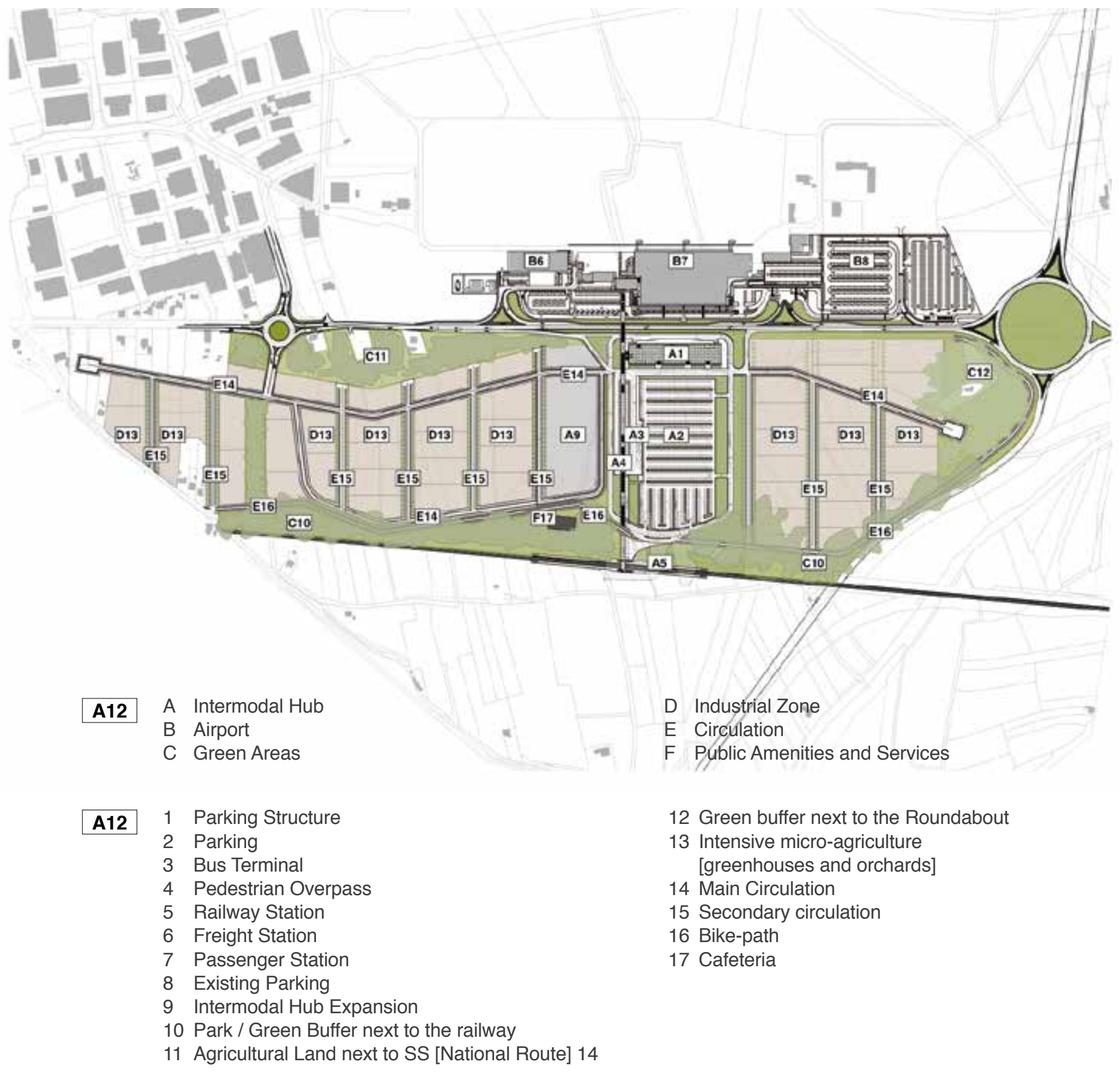

\subsubsection{Road System: Hierarchy and Types of Roads}

The vehicular handling system reproduces the layout proposed for the other scenarios, with the elimination of the penetration roads both in the West and East Sectors. Based on the need for greater interchange of freight traffic, it will be possible to create dedicated rail lines and an automated surface or underground link between the cargo section of the airport and the logistics area. Also the bicycle-pedestrian link 
follows the proposal made for the other scenarios, with a dedicated lane in the South of the area.

\subsection{Settlement Models}

\subsubsection{Main Functions, Induced Uses}

The Crisis Scenario is an evolution of the Optimal Scenario, therefore it starts from the same assumptions regarding the manufacturing/ production functions, combined with a significant presence of the logistics sector, bringing the area towards a situation of saturation and functional stress that does not allow a broader implementation of the planned functions. This hypothesis is configured as a case of success, which will require further development outside the scope of this research.

\subsubsection{Levels of Flexibility and Possibility of Modification}

In this scenario, the flexibility of the area is brought to a limit in terms of saturation and further evolution. Within the operating limits described above, the Sectors provide considerable implementation flexibility, with the creation of functional aggregates of various sizes up to the construction of two main buildings containing several related activities or large industrial or logistical plants.

\subsubsection{Land Use, Density, Green Areas, Economical Convenience and} Pragmatism

In this scenario, land use is brought to the maximum of its potential, while maintaining a distribution system capable of facilitating the densification of the built areas. The green areas, on the perimeter of the area, are confirmed. This in order to accommodate urban development standards in a concentrated number of locations, optimizing the design of the built areas, and to mitigate the impact of the production and logistics centre on the rest of the territory.

\subsection{Urban Layout and Contextualization of the Scenario}

\subsubsection{Stakeholders and Investors}

The main stakeholders of this scenario are entrepreneurs, both at the local and international level, able to harness the considerable potential offered by both the Intermodal Hub and the Friuli Venezia Giulia airport. The creation of a production and logistics system of such proportions could also offer the opportunity for the creation of an induced demand able to stimulate the creation of more companies operating in the production and service sectors. 
Figure 14. Sketches for the different scenarios.
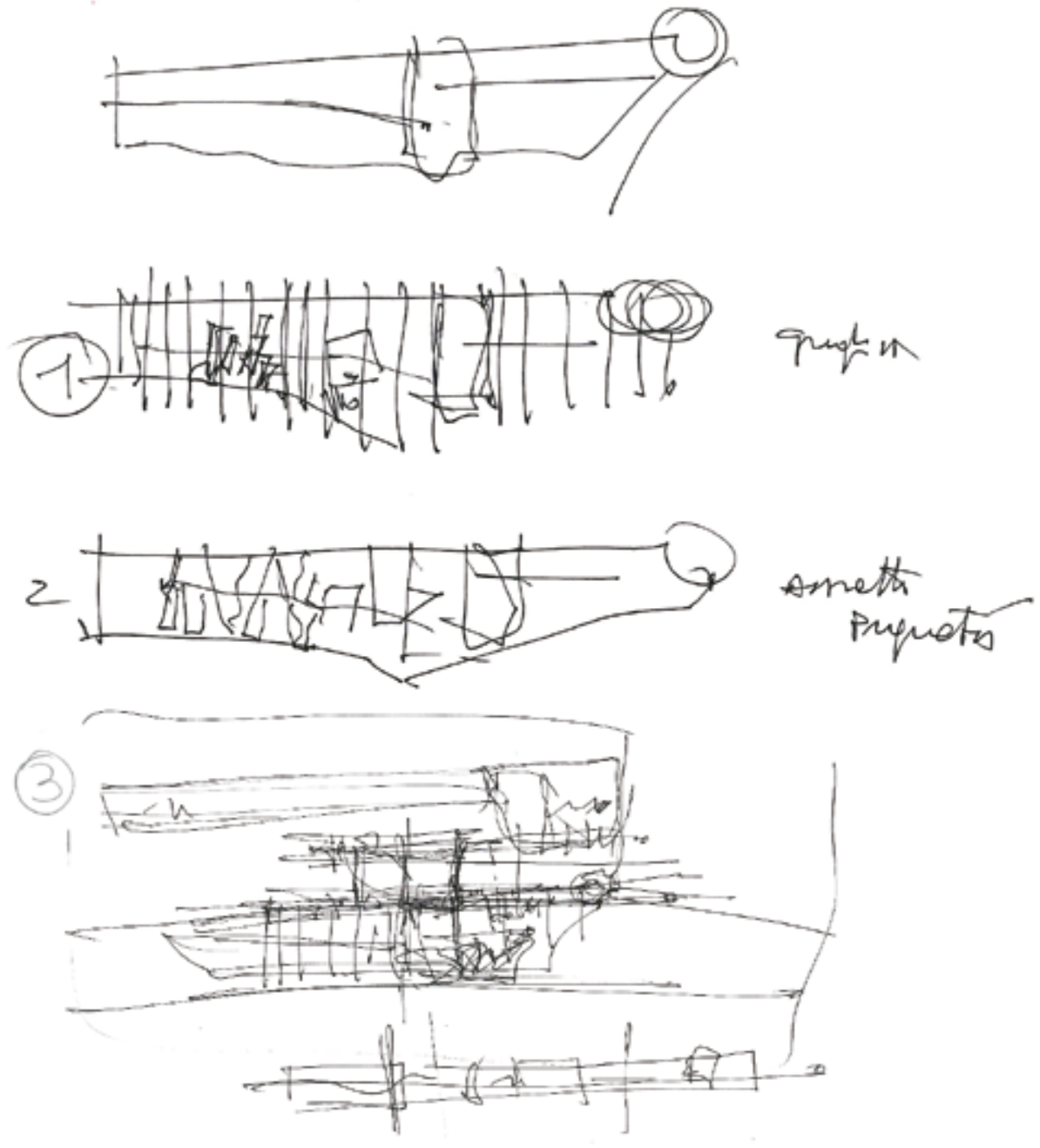

\subsubsection{Relations with the Intermodal Hub}

The Intermodal Hub is the backbone from which the road system of the two Sectors branches off, defining the building areas and their dedicated open spaces. Besides contributing to the formal definition and roadway layout of the production and logistics areas, the Intermodal Hub, integrated with an additional direct connection with the cargo section of the airport, is the engine of the logistics sector.

\subsubsection{Connections to the Context}

The connections to the surrounding area and the road system are mainly implemented through the ring of the Intermodal Hub. In addition to this primary link, there is a connection to the road system in the West of the area. To the South, the connection to the railway, which is strategic both in terms of passenger traffic related to the airport and commuter traffic to and from Trieste, plus a bicycle route connected to the local bicycle and pedestrian system, complete the whole infrastructure. 


\subsection{Conclusions}

The Crisis Scenario represents the maximum sustainable development for the area concerned by the study. The production and logistics activities benefit from the opportunities offered by the simultaneous presence of the airport and the Intermodal Hub, with a saturation logic beyond which it would be necessary to expand the facilities beyond the limits of the investigation carried out so far.

\section{GENERAL CONCLUSIONS}

At the conclusion of the three simulations, the study has found some characteristics and functional vocations in certain areas within the Sectors that undoubtedly can be defined as the invariants of the project. These are constituted by the filter areas on the perimeters of the Sectors, along the SS14 and along the Trieste-Venice railway line; the public green areas that structure the urban layout and the different settlement hypotheses; the main road system with "tangent rings" around the main ring of the Intermodal Hub; the general urban layout that identifies the Intermodal Hub as the centre of gravity of all possible solutions for development of the East and West Sectors. By contrast, it is possible to identify the system variables, mainly constituted by the areas that constitute the "heart" of the use foreseen for each scenario. It is believed that this aspect is important for the "success" of the proposed urban configurations, since it provides a considerable degree of freedom to the business initiatives and needs envisaged for each Sector. This high level of customization of the settlement model and the technical and productive organization will potentially boost the demand for settlement in the Sectors, if accompanied by incentives related to interventions that focus on rational land use, environmental quality and landscape qualification.

\section{Awards}

This essay was awarded Honorable Mention at the 2015 THE PLAN Best Paper Award contest. - Ed.

\footnotetext{
Note

The project of the Intermodal Hub of Ronchi dei Legionari was chosen as a finalist for the GLOBAL AIR RAIL AWARDS in Oslo in 2014 and in Toronto in 2015. Selected by an international panel of qualified experts in the field, it was one of the finalists for two of the nine categories of the event: Concept of the Year and Project of the Year, obtaining the second place in the final ranking. The Global Air Rail Awards reward the design and implementation of the best airport intermodal facilities in the world. The jury expressed its appreciation for the medium and long-term vision of the entire project, thanks to which "it will be possible to achieve positive results, both from the logistical and the environmental. Standpoints. Exploring in depth the strategic vision of the intervention, the project has highlighted its landscape characteristics and the definition of evolutionary scenarios that, offering a broad spectrum of potential uses of the adjacent sectors, will be able to adapt to different future economic conditions."
} 


\section{Acknowledgment}

This article is a result of a research activity carried out between September 2012 and January 2014 under an agreement between the University of Trieste, Department of Engineering and Architecture, and Aeroporto FVG S.p.A. of Ronchi dei Legionari on 10 May 2012.

Giovanni Fraziano, Architect, graduated at the IUAV in Venice with the highest honors where he taught Architectural Composition as researcher and Associate Professor until 2000. He was Visiting Professor in Nancy, Paris, Lausanne, Lyon and worked as Chief Editor of the architecture magazine Phalaris (1987-92). Since 2000, he has carried out teaching and research in the Faculty of Architecture in Trieste serving as Professor of Architectural and Urban Composition, as Dean (2009-2012), and currently as the Rector's Delegate for the construction and quality improvement of educational spaces and structures as well as coordinator of the Gorizia campus. In his awarded design activities, closely related to his academic experience, he addresses very heterogeneous and complex issues. E-mail: gfraziano@units.it

Thomas Bisiani, Architect, Ph.D., studied at the IFU (Paris), graduated in Venice in 2001 and received his Ph.D. in 2010 at the University of Trieste with mention of publication. He received an honorable mention at the Venice Biennale (2000); the Young Architects Award from the Italian Chamber of Deputies (2006); he won the Everyville competition and received the special award of the Biennale (2008); and coordinated "Piazza dell'Architettura," a festival dedicated to architecture in Trieste (2010-12). He is currently Lecturer at the University of Trieste. E-mail: bisianistudio@gmail.com

Luigi Di Dato, Architect, Ph.D., graduated with honors in Architecture at the IUAV of Venice (2003). He taught at the Faculty of Architecture of the University of Trieste, where he received his Ph.D. in Architectural and Urban Design (2009) and where he was Research Fellow (2012-13). Since 2004 he is engaged in project activities at the office Di Dato \& Meninno Architetti Associati. E-mail: didato@didato-meninno.com

Claudio Meninno, Architect, Ph.D., graduated with honors in Architecture at the IUAV in Venice (2003), studying also at the HUT (Helsinki) and attending the itinerant design seminar Villard de Honnecourt. He received his Ph.D. in Architectural and Urban Design at the University of Trieste (2009), where he taught as Research Fellow. He was visiting professor at the Faculty of Architecture in Ljubljana (2013-14) and he is currently Research Fellow at the Department of Engineering and Architecture of the University of Trieste. Since 2004 he is engaged in project activities at the office Di Dato \& Meninno Architetti Associati. E-mail: meninno@didato-meninno.com

Adriano Venudo, Architect, Ph.D., graduated at the IUAV in Venice in 2001. In 2007 he earned a Ph.D. in Architectural and Urban Design at the University of Trieste, specializing in the fields of infrastructure planning and mobility planning. He is lecturer at the Department of Engineering and Architecture of the University of Trieste and he has carried out design activities as a founding partner of the Stradivarie office. E-mail: avenudo@units.it

Marko Verri, Architect, Ph.D., graduated at the Faculty of Architecture in Trieste (2006) and obtained a Ph.D. in Architectural and Urban Design at the same University (2011). Professor of technology and techniques for the graphic representation at various state schools, he has collaborated in teaching and research activities at the Department of Engineering and Architecture of the University of Trieste. Since 2006, he has also carried out design activities by participating in various architectural contests.

E-mail: markoverri@gmail.com 